\title{
Novel avulsion pattern of the left principal bronchus with involvement of the carina and caudal thoracic trachea in a cat
}

\author{
Schmierer, Philipp A ; Schwarz, Andrea ; Bass, Danielle A ; Knell, Sebastian Christoph
}

\begin{abstract}
A 2-year-old, $4.5 \mathrm{~kg}$, neutered male domestic shorthair cat was presented to the emergency service with dyspnoea, anorexia and apathetic behaviour. Thoracic radiographs showed typical signs for a thoracic trauma and a tracheal lesion in the region of the carina consistent with pseudoairway formation. Computed tomography (CT) was performed in the conscious cat to avoid aggravation of air leakage associated with ventilation. The additional CT findings were consistent with a novel pattern of a traumatic avulsion of the left principal bronchus expanding into the carina and caudal thoracic trachea. Despite the complex avulsion pattern successful treatment was achieved surgically by performing an end-to-end anastomosis via a fifth right intercostal lateral thoracotomy. The cat was ventilated with a feeding tube and jet ventilation throughout. The cat showed excellent recovery 6 months after surgery.
\end{abstract}

DOI: https://doi.org/10.1177/1098612X13511623

Posted at the Zurich Open Repository and Archive, University of Zurich

ZORA URL: https://doi.org/10.5167/uzh-88186

Journal Article

Accepted Version

Originally published at:

Schmierer, Philipp A; Schwarz, Andrea; Bass, Danielle A; Knell, Sebastian Christoph (2014). Novel avulsion pattern of the left principal bronchus with involvement of the carina and caudal thoracic trachea in a cat. Journal of Feline Medicine and Surgery, 16(8):695-698.

DOI: https://doi.org/10.1177/1098612X13511623 
3 A novel avulsion pattern of the left principal bronchus with involvement of the carina and

4 caudal thoracic trachea in a cat

$5 \quad$ Philipp A Schmierer, Andrea Schwarz, Danielle A Bass, Sebastian Christoph Knell

6 Vetsuisse Faculty University of Zurich, Zurich, Switzerland

7 Philipp Schmierer med.vet, Vetsuisse Faculty University of Zurich, Winterthurerstr. 260, 8 Zurich, CH-8057, Switzerland, Email: pschmierer@vetclinics.uzh.ch

9

11 Summary

12 A two-year-old, $4.5 \mathrm{~kg}$, neutered male domestic shorthair cat was presented to the emergency 13 service with dyspnoea, anorexia and apathetic behaviour. Thoracic radiographs showed 14 typical signs for a thoracic trauma and a tracheal lesion in the region of the carina consistent 15 with pseudoairway formation. CT was performed in the conscious cat to avoid aggravation of 16 air leakage associated with ventilation. The additional CT findings were consistent with a 17 novel pattern of a traumatic avulsion of the left principal bronchus expanding into the carina and caudal thoracic trachea. Despite the complex avulsion pattern successful treatment was achieved surgically by performing an end-to-end anastomosis via a fifth right intercostal 20 lateral thoracotomy. The cat was ventilated with a feeding tube and jet ventilation throughout.

21 The cat showed excellent recovery six months after surgery.

\section{Keywords}

24 Bronchial avulsion, cat, pseudoairway

26 Intrathoracic tracheal avulsion is a rare but well described injury in the cat. ${ }^{1,2,3,4}$ 
27 A blunt trauma with hyperextension of the head and neck is assumed to be the initiating cause typically leading to an avulsion 1 to $4 \mathrm{~cm}$ cranial to the bifurcation of the trachea representing its weakest point. In some circumstances avulsion of a principal bronchus is possible. ${ }^{3,45}$ In the presented case a novel avulsion pattern was observed involving the bifurcation, the carina and the caudal trachea. After an avulsion injury, intact tracheal adventitia as well as thickening of mediastinal tissue is thought to maintain the airway lumen enabling ventilation leading to a condition described as -pseudoairway. ${ }^{3}$ However the narrowing at both avulsed ends of the injury can result in stenosis., ${ }^{2,3}$ Resection of the stenosed ends of the avulsed trachea followed by anastomosis is described as the treatment of choice. ${ }^{3,4,5,6}$ This case serves to report a completely novel avulsion pattern including its diagnostic work up on a conscious patient and its subsequent surgical repair.

A two-year-old, $4.5 \mathrm{~kg}$, neutered male domestic shorthaired cat was presented to the emergency service with dyspnoea, anorexia and apathetic behaviour for two days. A traumatic history was suspected. Tachypnoea and increased inspiratory lung sounds were present on the right side on auscultation, whereas lung sounds were diminished on the left side. Other vital parameters were within normal limits as well as serum biochemistry and haematology. Initially the cat was treated with intravenous Ringers Lactate solution (Ringer Lactate, Fresenius Medical Care AG\&Co.) at maintenance rate $(2 \mathrm{ml} / \mathrm{kg} / \mathrm{h})$ and buprenorphine (20 $\mu \mathrm{g} / \mathrm{kg}$ q6h Temgesic, Reckitt Benckiser). Thoracic radiographs revealed moderate pneumothorax, pleural effusion, pneumomediastinum and pneumoretroperitoneum. The base of the left mainstem bronchus appeared spherically enlarged and could not be followed to the periphery, raising the suspicion of an airway laceration (Figure 1).

Computed tomography was scheduled for the following day to assess severity and extent of 50 the lesion. Thoracocentesis was performed and examination of the fluid was consistent with the diagnosis of haemothorax. The cat was kept in an oxygen cage with continuous respiratory monitoring. 
53 Computed tomography was performed with a multi row helical CT unit (Brilliance16, Philips

54 AG) using 16 detector rows. Due to continuous concern regarding ventilation associated complications in anaesthesia, the study was performed with the animal awake in an appropriately sized conventional cardboard box, allowing oxygen supply and air circulation via small designated holes. The resulting plain scan was only minimally compromised by motion artefacts. Obliquity of patient positioning was corrected by adjusting the multiplanar reconstructions.

Scan direction was cranio-caudal using a slice thickness of $1.0 \mathrm{~mm}$. Scan parameters were $\mathrm{kV}$ 120, mA 183, collimator pitch 0.688 and 0.75 second tube rotation time. The field of view was adjusted to the cardboard box. The raw data was reconstructed to image series with $1 \mathrm{~mm}$ slices in a matrix of 512 dpi with an overlap of $33 \%$ in a soft tissue, bone and lung algorithm. The images were exported to a work station (OsiriX v.4.1. 64-bit, DICOM Viewer. See www.osirix-viewer.com) and multiplanar reconstructions were evaluated in appropriate windows (WL/WW): soft tissue (40/350), bone (300/1500) and pulmonary window ($500 / 1400)$.

The base of the left mainstembronchus was extensively enlarged over a length of $11.4 \mathrm{~mm}$, caudal to which it abruptly decreased in size with ill defined bronchial borders and tapered to the periphery. The left caudal lung lobe was aerated, whereas both parts of the left cranial lung lobe appeared isoattenuating to soft tissue, with only mild volume loss and persistent bronchial aeration. The right caudal mainstem bronchus was normal. Right lung lobe borders were mildly retracted. Moderate gas accumulations were visible around the mediastinal structures, expanding caudally into the retroperitoneal space and into subcutaneous and interfascial planes (Figure 2). The resulting diagnosis was rupture of the left mainstem bronchus, involving the carina, with secondary moderate pneumomediastinum.

77 Following intramuscular ketamine $(7 \mathrm{mg} / \mathrm{kg}$, Narketan, Vétoquinol), midazolam $(0.1 \mathrm{mg} / \mathrm{kg}$, Dormicum, Roche Pharma) and medetomidine (2 $\mu \mathrm{g} / \mathrm{kg}$; Dorbene), anaesthesia was induced 
with intravenous alfaxalone $(1.3 \mathrm{mg} / \mathrm{kg}$; Alfaxan, Vétoquinol) and maintained with alfaxalone (4.4-8 mg/kg/h), medetomidine $(0.5-1 \mu \mathrm{g} / \mathrm{kg} / \mathrm{h})$ and fentanyl $(5-10 \mu \mathrm{g} / \mathrm{kg} / \mathrm{h}$, Sintenyl, Sintetica SA) infusions. The trachea was intubated with a cuffed $4.5 \mathrm{~mm}$ endotracheal tube (Mallinckrodt, Covidien) just beyond the larynx. The cat was allowed to breathe spontaneously.

The bifurcation of the trachea was approached through a fifth right intercostal thoracotomy according to the description of White and Milner. ${ }^{3}$ A feeding tube (Paediatric Feeding Tube, Mallinckrodt, 5 FR) was advanced through the endotracheal tube lumen and connected to a manual jet ventilator (Manujet III, VBM Medizintechnik). Manual jet ventilation with driving pressures between $0.1-0.5$ bar, variable frequency and $\mathrm{FiO}_{2}$ of 1.0 was initiated thereafter. The effectiveness of ventilation was visually assessed by the degree of lung inflation.

The azygos vein was separated by blunt preparation and ligated. The caudal trachea as well as the bifurcation and the principal bronchi were isolated by a combination of sharp and blunt dissection and the oesophagus as well as the cranial vena cava were retracted. A pseudoairway was recognized in the area of the bifurcation. It was entered with a stab incision, which allowed observation of the two avulsed sites. At this state the feeding tube was advanced into the right bronchus under visual control. An oblique avulsion of the left principal bronchus with involvement of the carina and the distal trachea was detected (Figure $3)$.

When a decrease in arterial haemoglobin oxygen saturation and visual lung deflation occurred during opening of the pseudoairway membrane, the injured airway was digitally occluded. The proximal and distal end of the avulsed segment was transected in the cartilage as close as possible to the stenosed ends. Along the laceration the carina had to be resected to achieve proper anastomosis. Single interrupted 4-0 Polydioxanone (PDS, Ethicon, Johnson \& Johnson Medical $\mathrm{GmbH}$ ) sutures were pre-placed in the proximal and distal segment, starting at the dorsal aspect to facilitate best visibility. Most of the sutures were placed around 
cartilaginous rings. Due to the particular shape of the avulsion some of the sutures had to be placed through the end of cartilaginous rings. Mechanical ventilation was started after tying

107 the last suture and feeding tube removal. Warm sterile Ringers Lactate was used to lavage the 108 surgical site and to check for an airtight seal. A thoracic drain was placed, the thorax was 109 closed routinely and during closure a wound catheter (MILA Diffusion/Wound Catheter, 110 MILA International, Inc.) was placed for local bupivacaine $(0.5 \mathrm{mg} / \mathrm{kg} \mathrm{q} 6 \mathrm{~h}$, Carbostesin $1110.5 \%$, AstraZeneca AG) administration. Anaesthetic recovery was uneventful. Medetomidine 112 infusion (0.4-5.5 $\mu \mathrm{g} / \mathrm{kg} / \mathrm{h})$, buprenorphine $(20 \mu \mathrm{g} / \mathrm{kg} \mathrm{q} 6 \mathrm{~h})$ and intrapleural bupivacaine

$113(0.5 \mathrm{mg} / \mathrm{kg} \mathrm{q} 6 \mathrm{~h})$ administration was continued postoperatively.

114 The thoracic drain was removed 36 hours after surgery as thoracic radiographs showed no 115 signs of pneumothorax or persistent pseudoairway. The cat was discharged three days later. 116 Presentation at two weeks and four weeks after surgery showed the patient in a good 117 condition without clinical signs of respiratory abnormalities.

118 At telephone follow up six months after surgery, the owners reported a normal condition of 119 the cat without any signs of respiratory compromise.

120 A traumatic incident is considered to be the most common reason for avulsion injury of the 121 intrathoracic trachea ${ }^{4}$ and remains most likely in the presented case. A recent case report 122 described one report of an avulsion injury of a principal bronchus in a cat with a notional 123 traumatic episode, however in that case the bifurcation, the carina and the distal trachea 124 remained unaffected. ${ }^{5}$ The reason for the atypical and novel confirmation of the avulsion 125 injury described in this case report remains unclear. Similar injuries are described as avulsion 126 of the main bronchus with stellate tear in human medicine. The reason for this type of injury 127 is unexplained. ${ }^{7}$

128 The cat showed apathetic behaviour for two days prior to the time of presentation. White and 129 Milner described two distinct clinical presentations with the first to be an acute onset 130 dyspnoea and respiratory compromise immediately after the injury and the second a more 
131 chronic exertional dyspnoea combined with exercise intolerance appearing several days to

132 weeks after the traumatic episode. ${ }^{3}$

133 Thoracic radiographs initially had raised the suspicion of a discontinuity of the borders of the

134 left mainstem bronchus, resulting in a pseudoairway formation. The latter is described in 135 tracheal lacerations ${ }^{8}$, but is expected to develop in a similar manner in bronchial avulsions. ${ }^{4}$

136 Considering the aforementioned, the history of this cat is most consistent with a more chronic 137 avulsion injury.

138 The CT study allowed a distinct characterization of the bronchial lesion, hence facilitating 139 surgical and anaesthetic preparation. Being able to obtain a diagnostic study of a critical 140 patient in an awake state by creating a confined environment by such a simple device as a 141 cardboard box was beneficial.

142 The surgical approach via a right lateral thoracotomy to the tracheal bifurcation gave 143 excellent exposure as previously described. Left lateral thoracotomy would have restricted 144 the exposure by the descending thoracic aorta and the thoracic oesophagus. ${ }^{5}$

145 The authors conclude that avulsion injury of the principal bronchus with involvement of the 146 carina, the bifurcation and the distal trachea is a rare condition, which can be successfully 147 surgically repaired, even in cases with complex avulsion patterns. However it has to be taken 148 into consideration, that anaesthetic management of these patients is unpredictable and 149 challenging. Size and avulsion shape did not allow one-lung ventilation. ${ }^{9}$ Selective bronchus 150 intubation with subsequent jet ventilation was a feasible alternative ${ }^{9}$, although hypercapnia, 151 hypoxemia and barotrauma can occur. ${ }^{10}$

154 References

155 1. Ryan CO and Smith RA. Seperation of tracheal rings in a cat (What is your diagnosis?). 
158 2. Jörger K, Flückiger M and Geret U. Ruptur der Trachea bei drei Katzen. Berl Munch

159 Tierarztl Wochenschr 1988; 101: 128-131

160

161 3. White RN and Milner HR. Intrathoracic tracheal avulsion in three cats. J Small Anim Pract $1621995 ; 36: 343-347$.

163

164

4. White RN and Burton CA. Surgical management of intrathoracic tracheal avulsion in cats:

165 longterm results in 9 consecutive cases. Vet Surg 2000; 29: 430-435

166

167

168

5. White RN and Oakley MR. Left principal bronchus rupture in a cat. J Small Anim Pract 2001; 42: 495-498

169

170

6. Griffiths LG, Sullivan M and Lerche P. Intathoracic tracheal avulsion and

171 pseudodiverticulum following pneumomediastinum in a cat. Vet Rec 1998; 142: 693-696

172

173 7. Grover FL, Ellestad C, Arom KV, et al. Diagnosis and management of major

174 tracheobronchial injuries. Ann of Thorac Surg 1979; 28: 384-391

175

176 8. Zerenner DM, Agnello KA and Carberry CA. What is your diagnosis? Gas-filled spherical 177 dilatation of the trachea at the level of T3 through T5 vertebrae and narrowing of the trachea 178 lumen at T5. J Am Vet Med Assoc 2003; 12: 1683-1684

179

9. Mosing M, Iff I and Moens Y. Endoscopic removal of a bronchial carcinoma in a dog using 181 one-lung ventilation. Vet Surg 2008; 37: 222-225 
184 bronchial blocker of Univent during carinal resection. J Korean Med Sci 2010; 25: 1083-1085

185

186

187

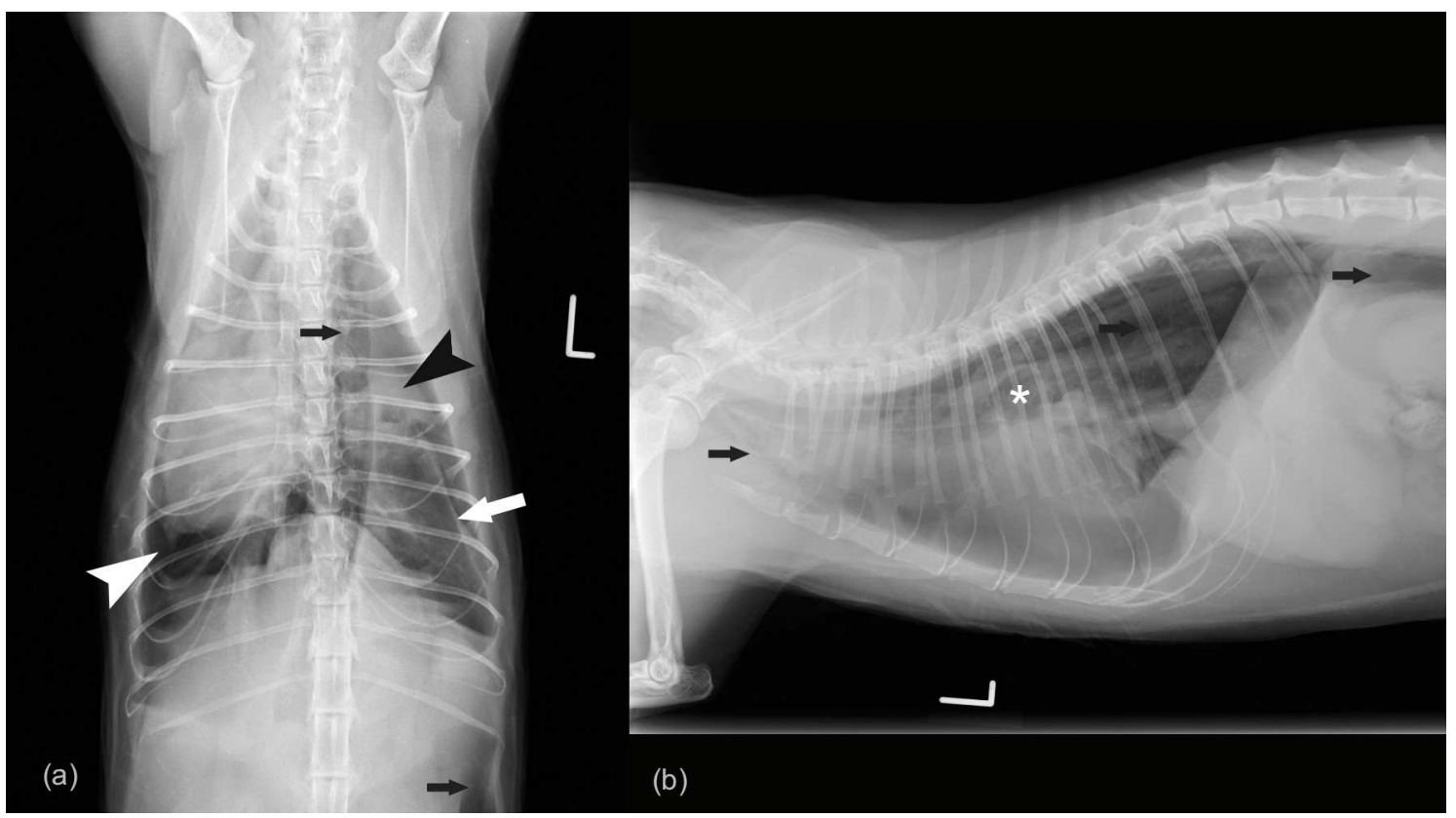

188

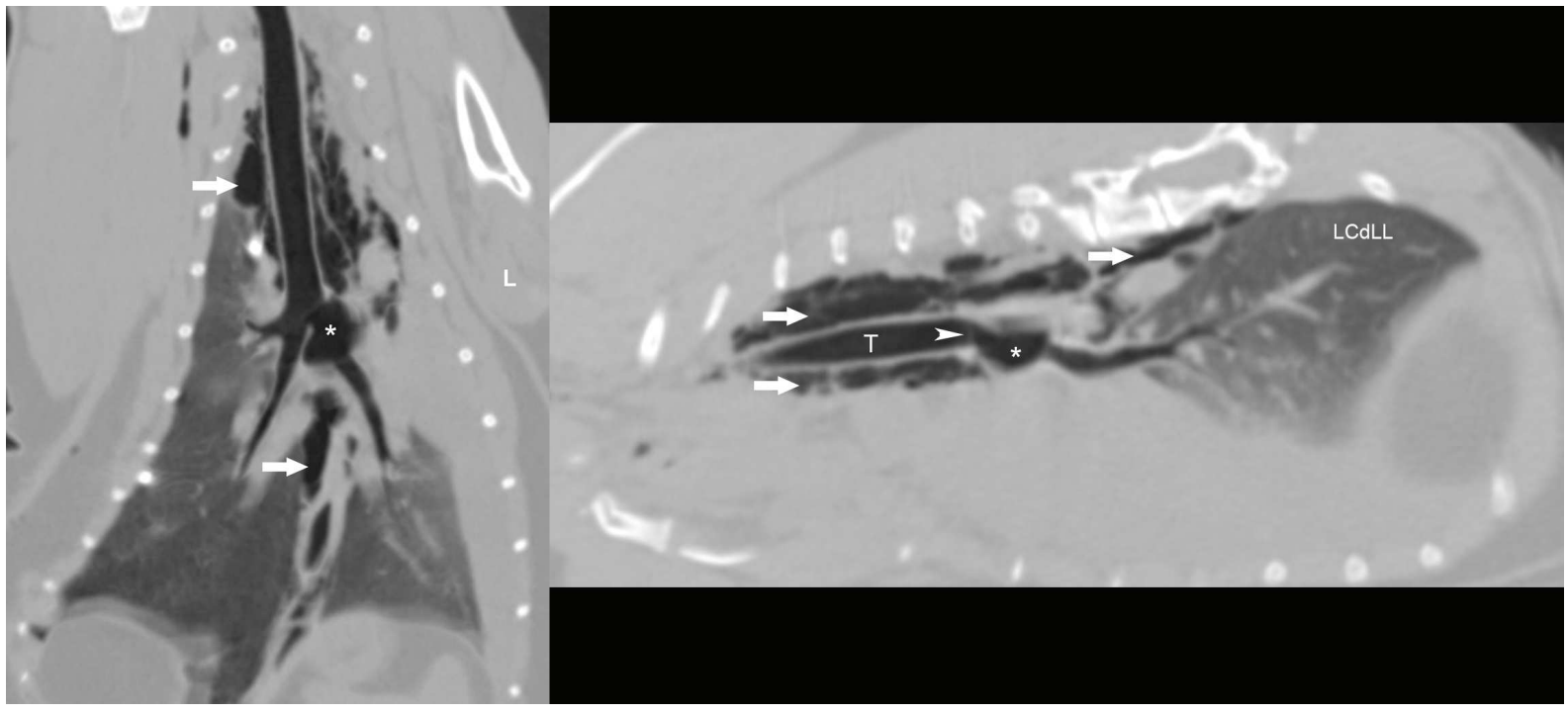

189

190

191

192

193

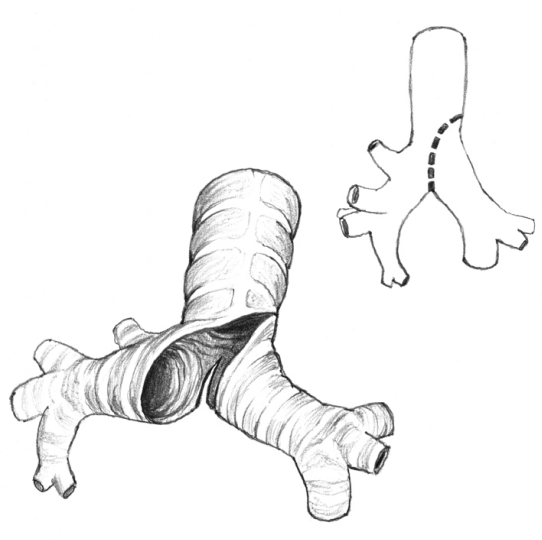

